\title{
The Early Pleistocene Equidae from Pirro Nord (Apricena, Southern Italy)
}

by

\author{
María Teresa Alberdi and Maria Rita Palombo
}

with 2 plates, 6 text-figures and 4 tables

\begin{abstract}
Fossil horse remains from Pirro Nord (Southern Italy) are described and compared with the late Early to Middle Pleistocene equids from the main sites of Western and Central Europe (Spain, France, Italy and Germany). Morphological features and dimensions indicate the unequal occurrence of two horses, close in morphology but different in size as supported by the statistical analyses (PCA and DA) performed including the most significant samples of Spain (Venta Micena, Barranco León-5, Fuente Nueva-3, Huéscar-1, Cúllar de Baza-1), France (Soleilhac), Italy (Venosa Loreto, Ponte Galeria), and Germany (Süßenborn, Untermaßfeld). The middle-sized horse is identified as Equus altidens altidens von Reichenau, 1915, its similarity with to the late early to Early Middle Pleistocene specimens from Guadix-Baza basin and Süßenborn is briefly discussed in order to chronologically assess the Pirro Nord "population". The second species Equus suessenbornensis WÜsT, 1900, poorly represented, is larger and close to the type population of Süßenborn, as well to the latest Early-early Middle Pleistocene large equids from South-Eastern Spain (Cúllar de Baza-1 and Huéscar-1), and Italy (Venosa Loreto).
\end{abstract}

Key words: Equus altidens, Equus suessenbornensis, Early Pleistocene, Pirro Nord, Italy

\section{Riassunto}

I resti degli equidi fossili di Pirro Nord (l'Italia Meridionale) sono descritti e comparati con quelli dei più importanti siti ad equidi del tardo Pleistocene inferiore e del Pleistocene medio inferiore dell'Europa Occidentale e Centrale (Spagna, Francia, Italia e Germania). Morfologia e dimensioni indicano la presenza di due cavalli, non molto distanti per morfologia ma chiaramente diverso per taglia. La presenza di due specie è confermata dai risultati delle analisi statistiche (PCA e DA) effettuate sul campione di Pirro e sugli esemplari affini per morfologia o all'incirca coevi di Spagna (Venta Micena, Barranco León-5 Fuente Nueva-3, Huéscar-1 il de di Cúllar Baza-1), Francia (Solilhac), l'Italia (Venosa Loreto, Ponte Galeria), e Germania (Süßenborn, Untermaßfeld). L'equide di taglia media è ascritta a Equus altidens altidens von ReICHENAU, 1915. Le affinità E. a. altidens di Pirro Nord e dei giacimenti del della fine del Pleistocene inferiore e del Pleistocene medio inferiore del bacino di Guadix-Baza (Huéscar e Cúllar de Baza-1) e del Pleistocene medio di Süßenborn e le relative implicazioni cronologiche sono brevemente discusse. L'equide di grande mole, Equus suessenbornensis WüsT, 1900, cui vanno ascritti un numero decisamente inferiore di resti, è morfologicamente e dimensionalmente confrontabile con la popolazione tipo del Pleistocene medio inferiore di Süßenborn, e con i resti della Spagna (Huéscar-1, Cullar Baza-1) e dell’ Italia (Venosa Loreto).

Parole chiave: Equus altidens, Equus suessenbornensis, Pleistocene inferiore, Pirro Nord, Italia

\section{Zusammenfassung}

Fossilisierte Pferde-Reste von Pirro Nord (Süd-Italien) werden beschrieben und mit Pferden des Oberen bis Mittleren Pleistozän aus den Hauptschichten West- und Zentraleuropas (Spanien, Frankreich, Italien und Deutschland) verglichen. Morphologische Merkmale und Abmessungen zeigen das ungleiche Ergebnis von zwei Pferden, die morphologisch sehr ähnlich sind, aber deutlich verschieden in der Größe. Diese Ergebnisse werden von statistischen Analysen (PCA und DA) unterstützt, die mit einer repräsentativen Probenauswahl Spaniens (Venta Micena, Barranco Léon-5, Fuente Nueva-3, Huéscar-1, Cúllar de Baza-1), Frankreich (Solilhac), Italien (Venosa Loreto, Ponte Galeria) und Deutschland (Süßenborn, Untermaßfeld) durchgeführt wurden. Das mittelgroße Pferd wird als

Addresses of the authors:

María Teresa Alberdi, Departamento de Paleobiología, Museo Nacional de Ciencias Naturales, CSIC, José Gutiérrez Abascal 2, 28006 Madrid, Spain, e-mail: malberdi@mncn.csic.es

Maria Rita Palombo, Dipartimento di Scienze della Terra, Università degli Studi di Roma “La Sapienza” and CNR- Istituto di Geologia Ambientale e Geoingegneria,Piazzale A. Moro, 5-00185 Rome, Italy, e-mail: mariarita.palombo@uniroma1.it 